\title{
An admission control algorithm for multi-hop 802.11e-based WLANs is
}

\author{
Yuxia Lin, Vincent W.S. Wong* \\ Department of Electrical and Computer Engineering, University of British Columbia, 2332 Main Mall, Vancouver, BC, Canada V6T $1 Z 4$
}

\section{A R T I C L E I N F O}

\section{Article history:}

Received 8 December 2007

Received in revised form 14 June 2008

Accepted 15 June 2008

Available online 26 June 2008

\section{Keywords:}

Admission control

WLANs

Contention graph

\begin{abstract}
A B S T R A C T
Recently, wireless local area network (WLAN) hotspots have been deployed in many areas. The new IEEE 802.11e standard further provides quality of service (QoS) provisioning by grouping the applications into four different access categories. The coverage area of WLANs can be extended by allowing the neighboring mobile devices to relay data to the access points. This concept is known as multi-hop WLANs or wireless mesh networks. Due to the limited network capacity and the contention-based channel access mechanism, admission control is required to regulate the number of simultaneous flows to maintain QoS. The multi-hop extension of WLANs present further challenges for admission control design due to the location-dependent contention in the network. In this paper, we propose an admission control algorithm for multi-hop 802.11e-based WLANs. In our proposed admission control algorithm, we first use a contention graph to model the contention situation in the multi-hop WLAN. It is followed by an estimation of the capacity for each maximal clique in the contention graph. A new flow is admitted if the aggregated traffic load is less than the estimated network capacity. The proposed algorithm supports both stationary and mobile nodes, as well as handoff and new connections. Simulation results show that the proposed algorithm is effective in providing QoS guarantee to the existing voice and video flows while maintaining a good performance for best effort traffic.
\end{abstract}

(c) 2008 Elsevier B.V. All rights reserved.

\section{Introduction}

The IEEE 802.11 wireless local area networks (WLANs) have gained extensive consumer markets for high-speed wireless Internet access. However, the current 802.11 medium access control (MAC) protocol does not provide quality of service (QoS) guarantee. Real-time applications may degrade their performance when the network becomes congested. With the increasing demand for multimedia services (e.g., voice calls, video streaming) over wireless, the newly approved IEEE $802.11 \mathrm{e}$ standard [2] provides a QoS extension to the current $802.11 \mathrm{a} / \mathrm{b} / \mathrm{g}$ WLANs. The Enhanced Distributed Channel Access (EDCA) mechanism in 802.11e, which is a direct extension to the Distributed Coordination Function (DCF), provides a contention-based QoS support.

In EDCA, each traffic flow is assigned to one of the four possible access categories (ACs). Each AC has a different medium access priority. It has been shown that this differentiation scheme works well when the network is moderately loaded [3]. However, due to the contention-based channel access, no strict QoS guarantee (e.g., throughput, delay) can be provided when the network be-

\footnotetext{
This paper was presented in part at the International Conference on Quality of Service in Heterogeneous Wired/Wireless Networks (QShine), Waterloo, Canada, August 2006 [1].

* Corresponding author. Tel.: +1 6048275135.

E-mail address: vincentw@ece.ubc.ca (V.W.S. Wong).
}

comes overloaded. For better QoS provisioning, resource management schemes such as admission control are essential to provide QoS guarantee. The QoS access point (QAP) can police the amount of traffic allowed into the network and maintain the network load so that it is below a certain threshold.

With the increasing popularity of the 802.11-based WLANs, there has been an interest in providing wireless Internet access via multi-hop WLANs or mesh WLAN networks [4,5]. In a multihop WLAN, one or more QAPs are connected to the Internet backbone. The mobile stations form an ad-hoc multi-hop network and relay the traffic to the QAP via each other (see Fig. 1). In densely populated areas, network access can be easily extended with this multi-hop structure without the cost of deploying more infrastructure devices [6]. This wireless mesh topology combines the advantages of WLAN's infrastructure working mode and the pure ad-hoc working mode. The network is both self-organizing and self-configuring. This leads to minimal upfront investment and ease of incremental installation.

Admission control for multi-hop WLANs is a challenging issue. For the single-hop WLAN case, all the QAPs and mobile stations experience similar medium access contention. The admission decisions are then based on the assumption that all the nodes can monitor the network conditions by some local measurements. However, for the multi-hop WLAN case, due to the location-dependent contention, the local medium usage information becomes ineffective to capture the traffic contention situation in the whole network. Thus, 


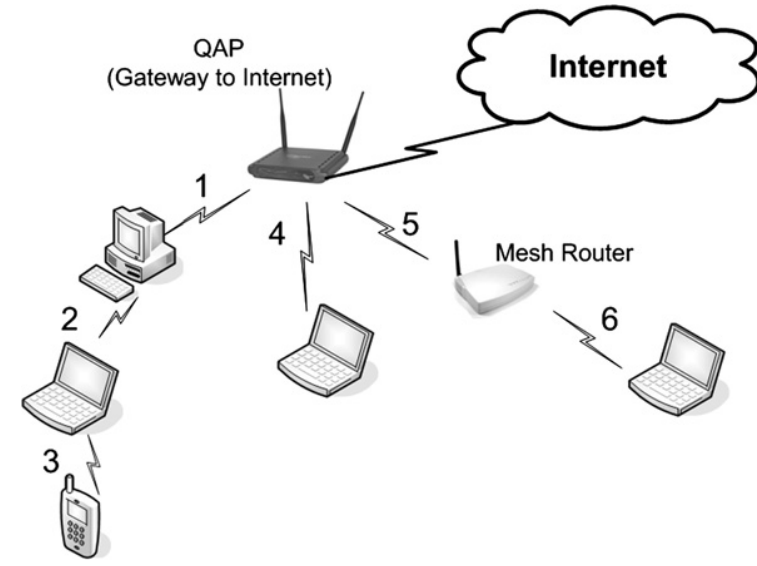

Fig. 1. A multi-hop WLAN. Each link is identified by an integer.

admission control algorithms proposed for the single-hop case cannot be directly extended to the multi-hop scenarios.

In this paper, we propose an effective admission control algorithm for multi-hop WLANs based on the IEEE 802.11e EDCA QoS extension. In our proposed admission control algorithm, we first use a contention graph (or conflict graph) to model the contention situation in the multi-hop WLAN. It is followed by an estimation of the capacity for each maximal clique in the contention graph via the use of the 802.11e saturation throughput analysis. A new flow is admitted if the aggregated traffic load is less than the estimated network capacity.

Node mobility is further considered in the system model. Handoff connections are given a higher priority in order to reduce the probability of dropping an on-going connection. Simulations are conducted under both linear and random topologies. Results show that the proposed algorithm is effective in providing QoS guarantee to the existing voice and video flows while maintaining a good performance for best effort traffic.

The rest of this paper is organized as follows. In Section 2, we provide an overview of the related new features in IEEE 802.11e and the related work in this area. In Section 3, we present the contention analysis for multi-hop WLANs and describe our proposed admission control algorithm for both stationary and mobile networks. Simulation results and discussions are presented in Section 4. Conclusions and future work are given in Section 5.

\section{Background}

In this section, we first describe the extension of the MAC protocol in the IEEE 802.11e standard. It is followed by a summary of various admission control algorithms.

\subsection{IEEE $802.11 e$}

The IEEE 802.11e standard [2] defines a Hybrid Coordination Function (HCF) to provide QoS guarantee. The HCF can use either a contention-based channel access method EDCA or a controlled channel access method HCCA (HCF Controlled Channel Access). EDCA is distributed and is compatible with the legacy DCF mechanism.

For EDCA, different traffic types are assigned to one of the four ACs. EDCA differentiates traffic priorities by assigning different sets of parameters to each AC. The parameters include the arbitration interframe space (AIFS), minimum and maximum contention window sizes $\left(C W_{\min }\right.$ and $\left.C W_{\max }\right)$, and transmission opportunity (TXOP).
This traffic differentiation works well in light to medium network load conditions. When the network load is high, increasing contention introduces excess collisions and retransmissions, which leads to a decrease in network throughput and an increase of delay [3]. To protect the existing traffic flows and provide QoS guarantee to the new flows, the IEEE 802.11e network can use admission control for resource management.

The IEEE 802.11e standard [2] defines the basic procedures for contention-based admission control for EDCA. A mobile station which needs to initiate a new real-time flow will first send an $A D D$ TS.request (Add-Traffic-Stream Request) frame to the QAP. The station specifies the traffic parameters such as the nominal MSDU (MAC Service Data Unit) size, mean data rate, and surplus bandwidth allowance in the ADDTS.request frame. Based on the requested traffic parameters and the network state, the QAP makes an admission decision, and sends back the results in the ADDTS.response frame. Note that the 802.11e standard only defines the signaling frame format for admission control. The network operators are free to implement their own admission decision algorithms. This paper proposes an admission control algorithm for EDCA-based multi-hop WLANs.

\subsection{Related work}

Due to the scarcity of wireless spectrum, radio resource management (RRM) is essential in QoS provisioning for wireless communication systems. RRM includes strategies and algorithms for wireless channel allocation, power control, modulation and coding schemes. Admission control is an important part of RRM, which grants or denies access of an arriving call to the network. The admission control decision is usually based on some predefined criteria, which depends on the network traffic condition, and the characteristics of the arriving call, such as its required network resources and whether it is a new or handoff call.

Admission control has been extensively studied for the wireless cellular networks in the past two decades [7]. For example, admission control for CDMA wireless networks has attracted particular interest due to its soft capacity limit. Call admission control is carried out to achieve a minimum signal-to-interference ratio (SIR). Another design criterion in wireless cellular networks is to control the handoff dropping probability in order to reduce the incidents of dropping an active call.

Admission control for WLANs has attracted more interest in recent years. Due to the probabilistic nature of the 802.11 medium access control, admission control for WLANs has many new challenging problems to solve. In [8], a distributed measurement-based admission control method is proposed for an 802.11 WLAN working in ad-hoc mode. Each mobile node measures the occupied bandwidth or the average collision ratio, and makes the admission decisions with a simple threshold rule. In [9-11], the expected network throughput or delay performance is estimated based on extensions to the Markov-chain model in [12] by applying the analytical model with measured parameters. An admission control decision is made at the QAP based on the new flow's request and the analytical prediction. However, it requires that admission control be applied to all four access categories, which may not be practical for bursty elastic data traffic such as HTTP (Hyper Text Transfer Protocol) sessions.

The work in [13] further extended the analytical modeling to 802.11e networks and proposed an admission control scheme by considering both bandwidth and delay performances. When a new call arrives, the expected collision probability is derived. The expected throughput and virtual time slot length are then calculated in order to compare them with the bandwidth and delay requirements of the incoming call. The new call is accepted when both throughput and delay requirements are met. A 
measurement-based admission control and dynamic bandwidth allocation scheme for the 802.11e HCCA function is proposed in [14] where the admission control is based on a threshold policy.

In [15], a distributed admission control scheme is proposed, which can be used in both infrastructure and ad-hoc modes. The QAP measures the medium utilization, and announces the transmission opportunity budget (TXOPBudget) via periodic beacon signals for each $A C$ (except $A C 0$ for best-effort traffic). When the TXOPBudget for one AC is depleted, new flows cannot gain transmission time and the existing flows cannot increase the transmission time either. In this case, the existing flows are protected. Several enhancements are further proposed in [3] by adjusting the contention level for data traffic.

The above admission control schemes are designed for the traditional single hop WLANs working under either infrastructure or ad-hoc mode. A reinforcement learning based admission control algorithm is introduced for a multi-hop mesh network in [16] where the admission objective is to maximize the network utility. This admission control scheme works well for a network with only best-effort traffic where the network operator's revenue can be maximized. But the admission control algorithm cannot provide QoS support for real-time flows because the throughput and delay performance is not guaranteed.

The work in [17] proposed a contention aware admission control protocol (CACP) to support QoS in multi-hop ad-hoc networks. Each node calculates the local available bandwidth by sensing the portion of the wireless medium time being idle. The available bandwidth in the carrier-sensing range neighborhood (c-neighborhood) is attained by either actively exchanging control frames or passively monitoring the channel. An admission control decision is made based on the bandwidth demand of the incoming flow and the available bandwidth at both the node and its c-neighbors. In [18], an admission control scheme for VoIP calls in a 802.11based wireless mesh network is proposed. However, the network capacity arising from multi-hop interference is estimated from simulation tests. The above schemes only work with one class of traffic for the 802.11-based ad-hoc networks. In our work, we aim to design an admission control algorithm that can support multiple classes of traffic with different QoS requirements in a multi-hop WLAN environment.

\section{System model}

In this section, we first describe the network model and the assumptions. It is followed by a discussion of the contention graph and maximal cliques. We then introduce our proposed admission control framework and describe how to estimate the capacity of each maximal clique by the saturation throughput analysis. We also describe the requirements to implement our proposed admission control algorithm in a QAP.

\subsection{Network model and assumptions}

Consider a multi-hop WLAN which is comprised of a set of $N$ nodes and a set of $L$ logical links. There is an access point which connects to the Internet and acts as the gateway for all the other mobile nodes in the network. Without loss of generality, we consider two ACs for EDCA. All the nodes use the same modulation scheme and transmission power, and are assumed to have the same receiver sensitivity performances. Thus, under similar propagation conditions, we can assume that every node has an identical transmission range $r_{\mathrm{tx}}$. The assumption of identical transmission range is widely adopted in the study of 802.11-based wireless networks [19-22]. One advantage of this simplified physical model is that the algorithms and protocols in the MAC and network layers can be more succinctly and clearly presented. We follow this simplified physical layer model to present the proposed admission control algorithm. Furthermore, a traffic flow subject to admission control usually keeps active from a few seconds to a few minutes, which is much longer than the time scale under which the wireless fading takes effect. As a result, it is suitable to consider the average link condition over a period of time for admission control purposes instead of the instantaneous transmission range under fading. If a device utilizes adaptive transmission power control, its transmission range will change over time due to power control. The proposed admission control framework can handle this situation as well. We simply need to update the varying transmission range to the access point periodically, which will introduce extra protocol overhead. But, if each node's propagation environment is different, the problem of accurately estimating each node's transmission range is beyond the scope of this paper.

A logical link exists between two nodes if the distance between them is less than or equal to $r_{\mathrm{tx}}$. The transmission from node $i$ to $j$ is successful if [23]:

1. There is a logical link between nodes $i$ and $j$.

2. No other nodes which are within the interference range $r_{\text {int }}$ of node $j$ transmit simultaneously.

\subsection{Contention Graph}

In general, the interference range $r_{\text {int }}$ is larger than $r_{\mathrm{tx}}$. The packets transmitted within the interference range may experience location-dependent contention for channel access. To capture the contention relations between different neighboring links in the network, we can construct a flow contention graph $G=\{V, E\}$ based on the above network model. In a contention graph, vertices correspond to the logical links. Each vertex $v \in V$ represents a logical link in the corresponding network graph. Given two logical links $u$ and $v \in V$, there is an edge $e_{u v} \in E$ if those two links are within the interference range of each other and they cannot be active simultaneously. As an example, Fig. 2(a) is a representation of the



(a) An example of multi-hop WLAN topology with 6 links.

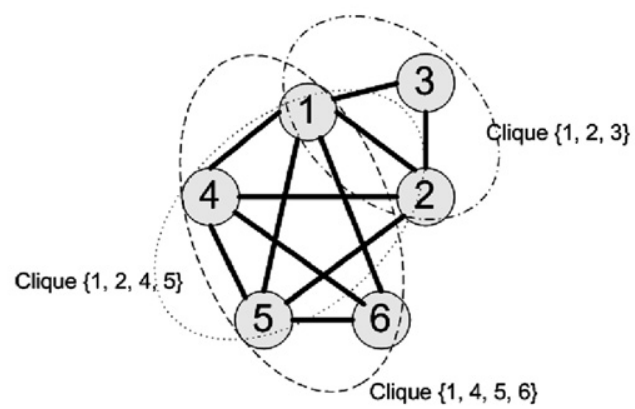

(b) The corresponding contention graph and maximal cliques.

Fig. 2. Contention graph and maximal cliques. 
network topology for the scenario in Fig. 1. The resulting contention graph is shown in Fig. 2(b).

In a contention graph, an induced subgraph which is complete is called a clique. In a clique, every vertex is adjacent to every other. A maximal clique is a clique which does not belong to any other larger cliques. For example, in Fig. 2(b), there are three maximal cliques: links $\{1,2,3\}$, links $\{1,2,4,5\}$, and links $\{1,4,5,6\}$. All the links in each maximal clique share the same channel resources and thus only one of them can be active at any given time. However, links which belong to different maximal cliques can be active simultaneously.

\subsection{Contention Analysis of 802.11e WLAN}

Consider a contention graph which has $M$ maximal cliques. We define the contention matrix $\mathbf{F}$ as:

$\mathbf{F}_{m l}= \begin{cases}1, & \text { if link } l \in \text { clique } m, \\ 0, & \text { otherwise }\end{cases}$

The dimension of matrix $\mathbf{F}$ is $M \times L$.

Suppose there are in total $S$ traffic flows between mobile nodes and the QAP. We define the routing matrix $\mathbf{R}$ as follows:

$\mathbf{R}_{l s}= \begin{cases}1, & \text { if flow } s \text { uses link } l, \\ 0, & \text { otherwise. }\end{cases}$

The dimension of matrix $\mathbf{R}$ is $L \times S$.

For the $S$ flows in the network, we assume that they belong to one of the two access categories (i.e., $A C_{1}$ or $A C_{2}$ ). We define the source rate vector for $A C_{i}$ as:

$\boldsymbol{x}_{\boldsymbol{i}}=\left[x_{i 1}, x_{i 2}, \ldots, x_{i S}\right]^{\mathrm{T}}, \quad i=1,2$

where $x_{i j}$ is the source rate of flow $j$ in $A C_{i}$.

The traffic load from $A C_{i}$ on each link can be calculated as:

$\boldsymbol{y}_{\boldsymbol{i}}=\mathbf{R} \boldsymbol{x}_{\boldsymbol{i}}=\left[y_{i 1}, y_{i 2}, \ldots, y_{i L}\right]^{\mathrm{T}}, \quad i=1,2$

where $y_{i l}$ is the total traffic load on link $l$ from $A C_{i}$ traffic.

The traffic load from $A C_{i}$ on each maximal clique is:

$\boldsymbol{z}_{\boldsymbol{i}}=\mathbf{F} \boldsymbol{y}_{\boldsymbol{i}}=\left[z_{i 1}, z_{i 2}, \ldots, z_{i M}\right]^{\mathrm{T}}, \quad i=1,2$

A new flow can be accepted only when the total traffic load on each maximal clique does not exceed the available capacity $\boldsymbol{c}_{\boldsymbol{i}}$ in that clique. That is,

$\boldsymbol{z}_{\boldsymbol{i}} \preceq \gamma_{i} \boldsymbol{c}_{\boldsymbol{i}}, \quad i=1,2$

where $0<\gamma_{i}<1$ and is a tunable parameter.

The challenge presented by the above admission decision framework is how to obtain an accurate estimation of the capacity $\boldsymbol{c}_{\boldsymbol{i}}$ in each maximal clique. On a wireline communication link, the channel capacity is usually well defined because the communication channel is a dedicated link between one transmitter and one receiver. On the other hand, for wireless networks, the channel capacity can be interpreted in different ways. Some may view it as the physical layer transmission rate which is a fixed value given the transmission power, coding and modulation schemes. However, in a CSMA/CA based WLAN, the throughput achieved by each node usually falls short of the physical transmission rate due to the protocol overhead and the contention to the shared transmission medium.

Thus, it is appropriate to define the wireless channel capacity as the attainable throughput above the MAC layer, which is similar to the definition used in [24]. For example, in an IEEE 802.11 network, the achievable system throughput is usually less than $80 \%$ of the physical transmission rate [12]. And this value varies with the number of stations in the network and the selected MAC parameters. Thus, in this paper, we use the achievable throughput as the channel capacity instead of the nominal physical transmission rate.

\subsection{Available capacity estimation}

The determination of the capacity of multi-hop WLANs is a nontrivial task. Information theory may provide a general bound of the achievable capacity [24-26]. However, the assumptions used in those models restrict their applicability to provide an accurate capacity estimation in 802.11-based multi-hop WLANs. To this end, we use the concept of saturation throughput in a single hop WLAN and extend it to the multi-hop case.

In a single-hop WLAN, the saturation throughput is defined as the throughput limit reached by the network in an overload condition (i.e., each node always has data packets to send) [12]. To study the effectiveness of using the saturation throughput as a capacity measure, a simple ns-2 simulation experiment is run for an 802.11b WLAN with ten mobile stations. Each station generates constant bit rate (CBR) traffic flow and the data packet size is 1500 bytes. Fig. 3 shows the measured network throughput as a function of the offered traffic load. We can observe that the network throughput increases linearly with the offered load until it reaches the maximum throughput. Afterwards, the network enters the overload state and the throughput remains at the saturation throughput level, which is slightly lower than the maximum throughput. As a result, the saturation throughput is a good performance figure of the system. It represents the maximum load that the system can carry in stable condition.

Recall that $\boldsymbol{c}_{\boldsymbol{i}}=\left[c_{i 1}, c_{i 2}, \cdots, c_{i M}\right]^{\mathrm{T}}$ denotes the capacity vector of AC $i$. The element $c_{i m}$ denotes the capacity (i.e., saturation throughput) of maximal clique $m$ for $A C_{i}$. Given the set of active nodes in each maximal clique $m \in M$, the saturation throughput is used as the achievable throughput estimate for $c_{i m}$. There are several analytical models for saturation throughput determination in the literature (e.g., [27-30]). We use the one proposed in [31]. Let $N_{i m}(i=1,2)$ denote the number of nodes with active $A C_{i}$ traffic in maximal clique $m$. From [31], we have

$c_{i m}=r_{s i, m} T_{p, i}, \quad i=1,2, \quad m=1,2, \ldots, M$

where $T_{p, i}$ is the time used to transmit the frame payload information for an $A C_{i}$ packet, and

$r_{s 1, m}=K_{m}\left(1-p_{1}\right)\left[\left(1-\frac{p_{1}}{2}\right) K_{m} T_{1}+\left(1-\frac{p_{2}}{2}\right) T_{2}\right]^{-1}$,

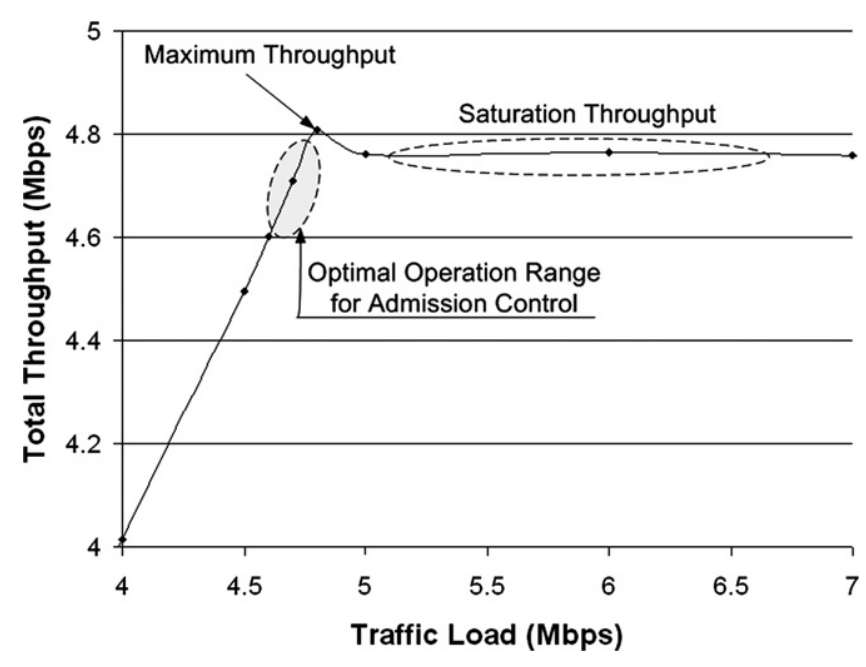

Fig. 3. Network throughput with increasing traffic load. 
$r_{s 2, m}=\left(1-p_{2}\right)\left[\left(1-\frac{p_{1}}{2}\right) K_{m} T_{1}+\left(1-\frac{p_{2}}{2}\right) T_{2}\right]^{-1}$,

$K_{m}=\frac{N_{1 m} \tau_{1}}{N_{2 m} \tau_{2}}$

where $\tau_{i}$ denotes the probability for an $A C_{i}$ packet to transmit in an idle time slot, $N_{i m}$ denotes the number of nodes in maximal clique $m$ that have AC $i$ traffic to send, $p_{i}$ denotes the packet collision probability of an $A C_{i}$ packet, and $T_{i}$ is the time required to finish a transmission cycle for $A C_{i}$ traffic. The equations required to obtain the numerical solutions for the above variables can be found in [31].

The saturation throughput is the throughput achieved when all $N_{i m}$ nodes in the maximal clique $m$ always have AC $i$ traffic to send. This can be a good estimation of the capacity of a clique. Since the saturation throughput is slightly lower than the maximum throughput which the network may achieve, this can also be viewed as a conservative estimation of the capacity of a clique. However, for admission control decisions, a conservation approach can always ensure better QoS guarantee.

\subsection{Admission control algorithm}

We now describe how to implement our proposed admission control algorithm at the QAP. We assume that all wireless stations are stationary and each station has access to some location service which can provide the geographical coordinates. For outdoor applications, GPS is nowadays readily available. In addition, different localization techniques can be used in our work, as presented in recent research papers [32,33], etc. Discussion about location services is out of scope of this paper. The position information is sent to the access point in the initial association process. When a wireless station initializes a new flow, it sends an ADDTS.request frame to the QAP. When the QAP receives the request frame, it updates the contention matrix $\mathbf{F}$, the routing matrix $\mathbf{R}$ and the source rate vector $\boldsymbol{x}_{\boldsymbol{i}}$ (by assuming that the new flow is accepted). It then computes the expected traffic load $z_{i}$. The QAP then calculates the expected saturation throughput in each maximal clique and uses it to construct the maximal cliques' capacity vector $\boldsymbol{c}_{\boldsymbol{i}}$. The new flow will be accepted only if Eq. (6) is satisfied. Otherwise, the new flow will be rejected. Finally, the QAP will notify the mobile station its decision via the ADDTS.response frame. The admission control algorithm is summarized in Algorithm 1.

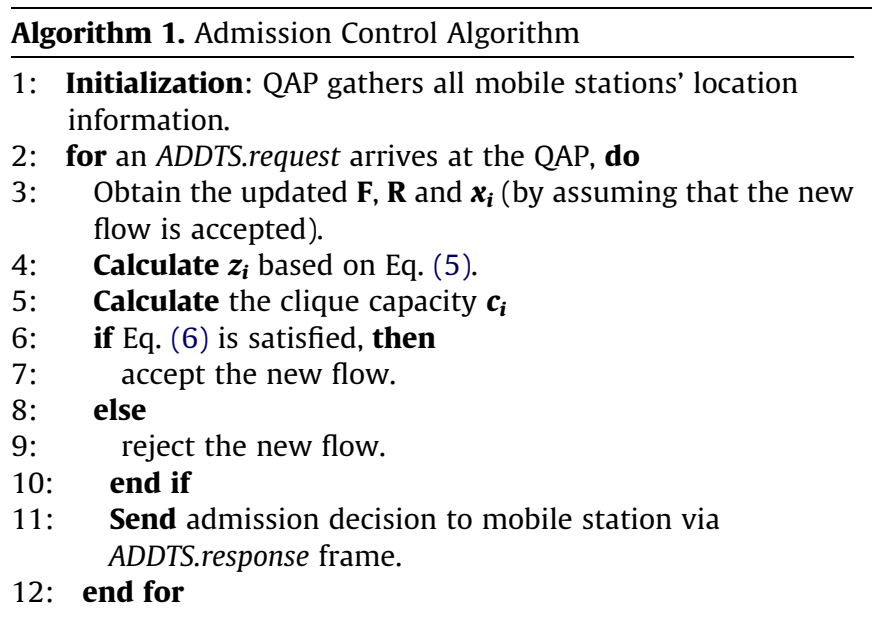

The above algorithm works with stationary stations. Extension to mobile nodes will be discussed in Section 3.7.

\subsection{Extension for best-effort traffic}

The algorithm described in the previous section assumes that both $A C_{1}$ and $A C_{2}$ traffic flows are subject to admission control. However, data traffic are usually bursty in nature and may not be suitable for admission control. Instead, one needs to protect those best effort flows so that the event of bandwidth starvation will not occur. Our proposed algorithm can be extended to handle the case of best-effort traffic.

Without loss of generality, we assume that $A C_{1}$ is subject to admission control and $A C_{2}$ is for best-effort traffic. The active links used by the best-effort traffic flows will still be utilized for constructing the contention graph. In addition, the number of active $A C_{2}$ nodes in each clique will also be used for clique capacity estimation. This usually leads to a conservative estimation of the channel capacity for the real-time flows, because not all $A C_{2}$ nodes may be active simultaneously. Furthermore, the best-effort traffic such as TCP flows have congestion control mechanisms and usually will not cause the network to be operated in a saturated condition. As a result, the admission control Algorithm 1 can be used with the following changes:

1. The source rate vector in Step 3 will only include $A C_{1}$.

2. For the decision making in Step 6 , the constraint will only be checked for $A C_{1}$.

In this case, the value of $\gamma_{1}$ can be adjusted according to the amount of network capacity allocated for best traffic. For example,

Table 1

IEEE 802.11e parameters

\begin{tabular}{ll}
\hline Basic Rate & $1 \mathrm{Mbps}$ \\
Data Rate & $11 \mathrm{Mbps}$ \\
PLCP preamble and header & $192 \mathrm{bits}$ \\
MAC header + FCS & 224 bits \\
ACK frame size (excluding PLCP) & 112 bits \\
Time slot & $20 \mu \mathrm{s}$ \\
SIFS & $10 \mu \mathrm{s}$ \\
\hline
\end{tabular}

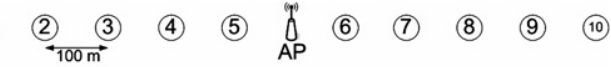

(a) Linear Topology.

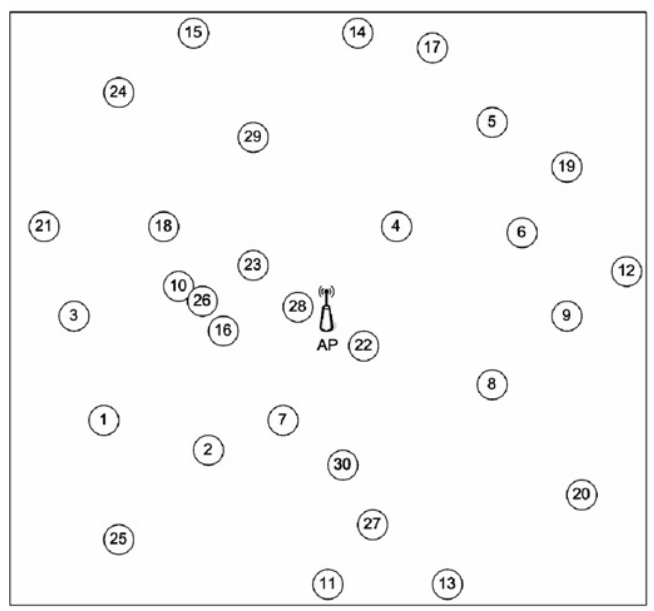

(b) Random Topology.

Fig. 4. Simulation Topologies. 
a small $\gamma_{1}$ in Eq. (6) will provide more bandwidth for best-effort traffic.

\subsection{Extension for mobile user terminals}

The above analysis was based on the assumption that the network topology is stationary. However, it is not difficult to extend it to account for mobile users in the multi-hop network. This section describes the extensions needed to accommodate node mobility. The particular concern to investigate with mobility is the handoff process where a mobile node may change its route to the QAP due to its own movement or movement of any of the intermediate relaying nodes. We assume that the location information of each mobile node is periodically updated to the QAP, such that the QAP may have relatively accurate location information for the admission control decision.

When user terminals move around, the change in distances between nodes may alter the contention relationships between them, which in turn changes the contention matrix F. Moreover, when two nodes move beyond the transmission range of each other, the wireless link between them may break. Any existing route utilizing this link will become invalid and a route discovery process will be initiated to find a new route by an ad-hoc routing protocol. This will lead to a change in the routing matrix $\mathbf{R}$. Both $\mathbf{F}$ and $\mathbf{R}$ are important parameters for making the correct routing decision in the admission control algorithm. When $\mathbf{F}$ and $\mathbf{R}$ change, the QoS requirement of some of the accepted calls may no longer be satisfied, and those calls may have to be dropped. Since the interference range is generally much larger than the transmission range, route changes in $\mathbf{R}$ occur more often than changes in the contention graph $\mathbf{F}$, and has a greater impact on system performance. As a result, we may only consider the route change events. When a mobile node switches from an old routing path to a new one, we may consider this to be equivalent to the horizontal handoff process in a wireless cellular network where the user terminal switches from one base station to another.

For new calls from a mobile station, the admission process can be carried out the same as specified in Algorithm 1. However, when the route between a mobile terminal and the QAP is changed due to node mobility and a new route is established at the mobile node, a ADDTS.renew frame will be sent to the QAP requesting that the existing QoS flow be handed over to the newly established path. Upon receiving the ADDTS.renew frame, the QAP will update the $\mathbf{F}$ and $\mathbf{R}$ matrices using the updated information, and will decide whether to accept the handoff call based on:

$\boldsymbol{z}_{\boldsymbol{i}} \preceq \gamma_{i}^{\prime} \boldsymbol{c}_{\boldsymbol{i}}, \quad i=1,2$

where $0<\gamma_{i}^{\prime}<1$ is a tunable parameter generally greater than $\gamma$ in (6), such that the handoff call has a higher priority than the new calls in order to reduce the probability of dropping an on-going call. The algorithm is summarized in Algorithm 2.

\section{Performance evaluation}

To verify the effectiveness of our proposed admission control algorithm, we perform simulation experiments by using ns-2 [34]. The IEEE 802.11 MAC parameters used are shown in Table 1. The transmission range of each wireless station, $r_{\mathrm{tx}}$, is $100 \mathrm{~m}$. The interference range, $r_{\text {int }}$ is twice the transmission range. Only basic access scheme is used, and no channel error is considered. We first consider the cases when only real-time traffic is present in Sections 4.1 and 4.2. Section 4.3 investigates the cases when both real-time and best-effort traffic are present. Section 4.4 studies the performance under node mobility.
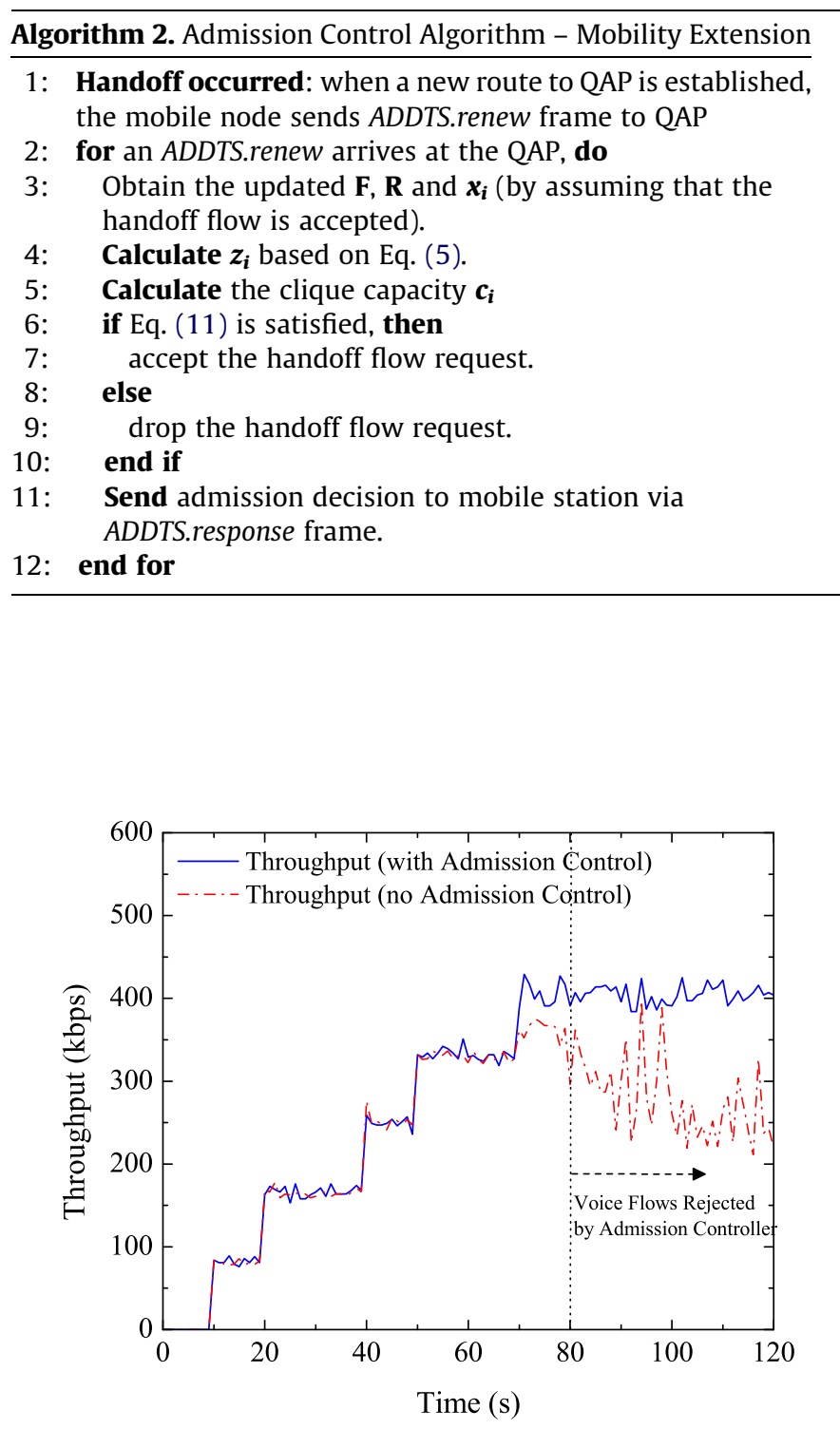

(a) Throughput.

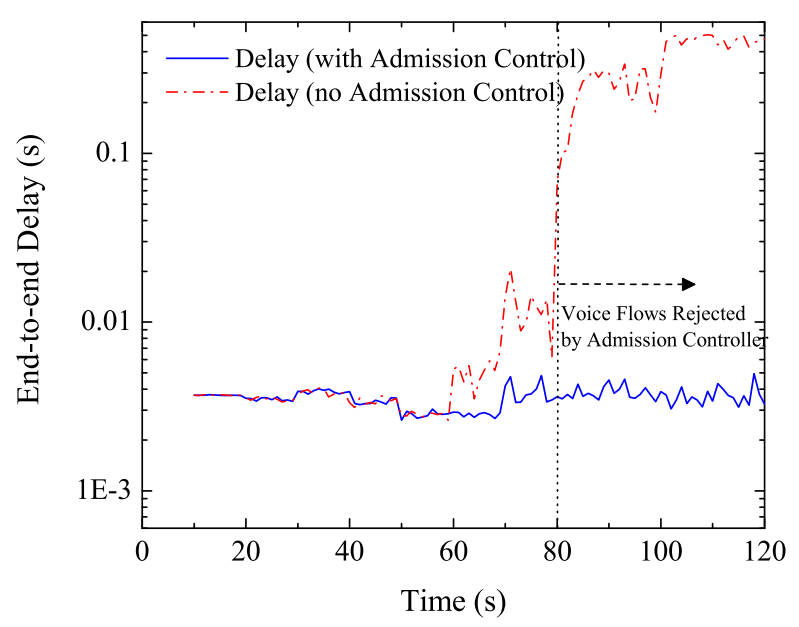

(b) Delay.

Fig. 5. Performance of voice flows under linear topology. 


\subsection{Linear topology}

We first examine the performance of the proposed admission control scheme under a linear topology with 10 mobile stations, as shown in Fig. 4(a). The distance between any two neighboring stations is $100 \mathrm{~m}$. Real-time traffic flows (either voice or video) arrive at each mobile station periodically. These flows are subject to the admission control algorithm proposed in the previous section. Each voice flow is a CBR source with a fixed payload size of 208 bytes (i.e., 160 bytes G.711 payload + RTP/UDP/IP/LLC/SNAP Headers) and an inter-arrival time of $20 \mathrm{~ms}$. It corresponds to an $83.2 \mathrm{kbps}$ traffic stream.

Each video traffic source is a CBR data source, using UDP with a constant payload of 1500 bytes and an average inter-arrival time of $40 \mathrm{~ms}$. It corresponds to a $300 \mathrm{kbps}$ traffic stream. The AIFSN and $C W_{\min } / C W_{\max }$ values are set according to the IEEE 802.11e standard [2]. $C W_{\min / \max }$ for AC_VO (Voice flow) is $7 / 15$, and for AC_VI (Video flow) is $15 / 31$. AIFSN for AC_VO and AC_VI are both equal to 2 . The Destination-Sequenced Distance-Vector Routing (DSDV) [35] is used as the routing protocol.

At time $10 \times i$ second, mobile station number $i$ initiated a flow towards the QAP by sending an ADDTS.request frame to the QAP.

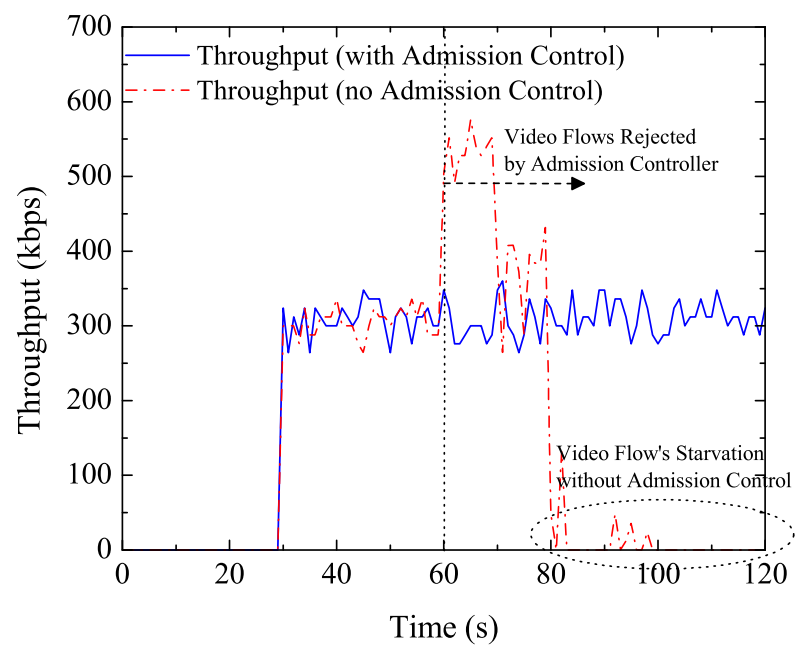

(a) Throughput.

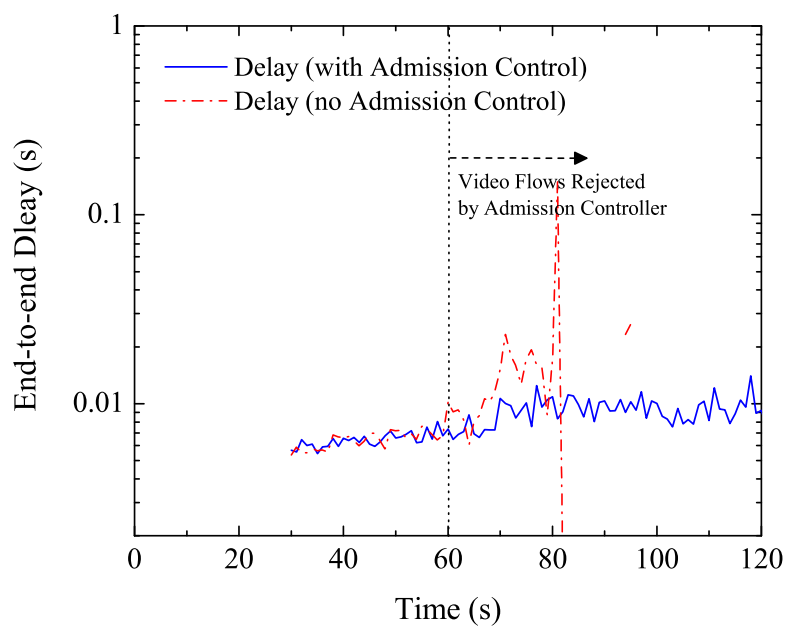

(b) Delay.

Fig. 6. Performance of video flows under linear topology.
It is either a video flow when $i$ is a multiple of 3 , or a voice flow otherwise. Thus, the video and voice flows arrive alternatively. The QAP either accepts or rejects the flow request based on the decision rule in Eq. (6). A value of 0.85 is used for both $\gamma_{1}$ and $\gamma_{2}$.

Results for the throughput and delay performance of voice flows are shown in Fig. 5. The results for video flows are shown in Fig. 6. We compare with the case when no admission control mechanism is enforced. From these figures, we can observe that the admission control algorithm begins to reject the video flows at time $60 \mathrm{~s}$, and to reject the voice flows at time $80 \mathrm{~s}$. In this way, the network protects the existing voice and video flows that have been admitted to the network. All the admitted real-time flows have an end-to-end delay to be below $10 \mathrm{~ms}$. Without admission control, the delay begins to increase significantly from time $60 \mathrm{~s}$ onwards, and the throughput begins to decrease due to packet loss.

\subsection{Random topology}

In the second simulation, 30 mobile stations are randomly distributed in a $500 \mathrm{~m} \times 500 \mathrm{~m}$ coverage area, with the QAP located at the center as shown in Fig. 4(b). The mobile stations are numbered from 1 to 30 consecutively. At time $10 \times i$ second, station number $i$

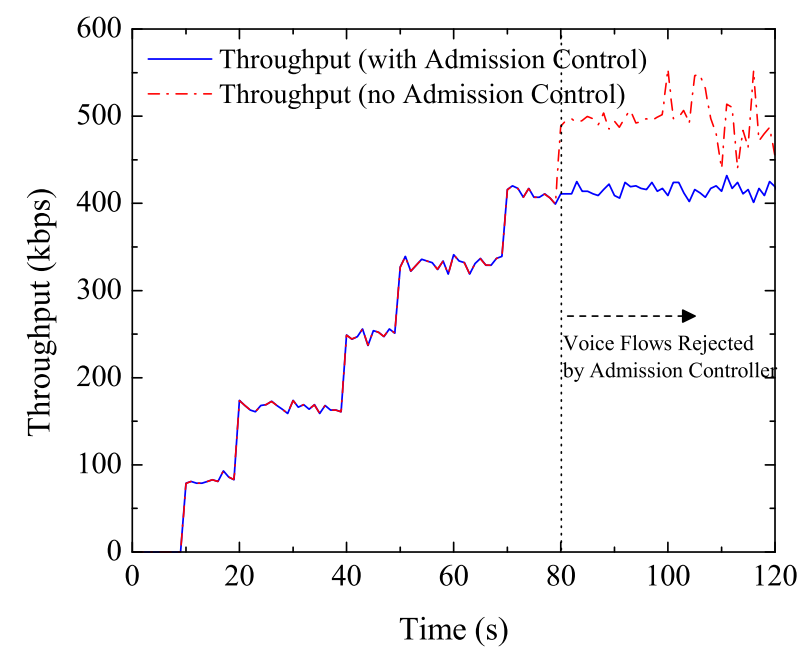

(a) Throughput.

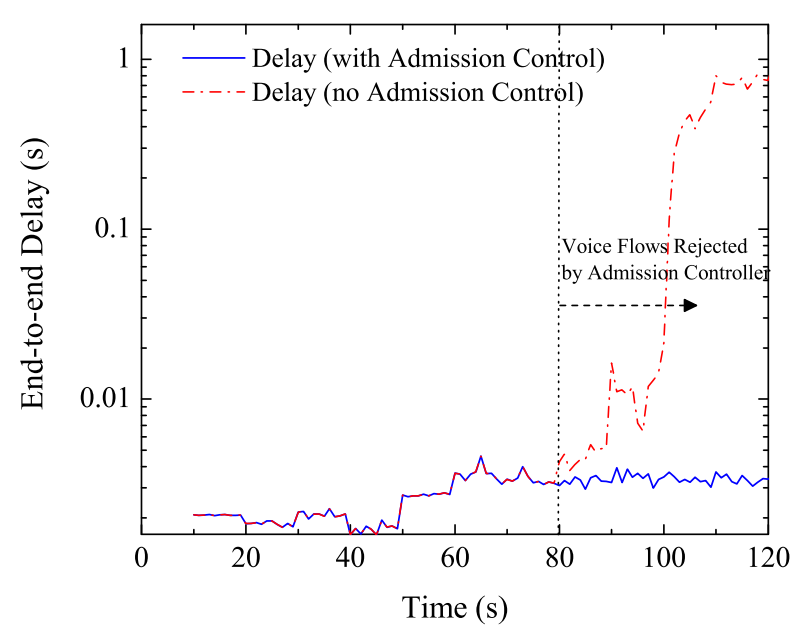

(b) Delay. 
initiates a flow to the QAP. It is either a video flow if $i$ is a multiple of 3 , or a voice flow otherwise. All other parameters are the same as in the linear topology test.

The throughput and delay performance for voice and video flows are shown in Figs. 7 and 8. The results show that the admission control algorithm rejects voice flows from time $80 \mathrm{~s}$, and video flows from time 90 s onwards. It accepts one more video flow than the linear topology. This is expected, as the two dimensional space provides better spatial separation between flows than the one dimensional linear topology, which leads to less contention and higher capacity in the network.

\subsection{Real-time and best-effort traffic flows}

In this test, we examine the effectiveness of the admission control algorithm when both real-time and best-effort traffic co-exist. The test uses the same random topology as in the previous simulation. FTP and voice flows use the AC_VI and AC_VO EDCA parameters as specified in Section 4.1, respectively. The stations numbered 3, 6, 9 initiate three FTP flows to the QAP via TCP connections at time $10 \mathrm{~s}$. Afterwards, voice flows arrive every $10 \mathrm{~s}$ from station numbered $1,2,4,5,7,8,10,11$, and 12 . We do not perform admis- sion control on the FTP flows. For the voice flows, we set $\gamma_{1}=0.6$. The throughput and delay performance for voice flows are shown in Fig. 9. The performance of FTP flows is shown in Fig. 10. We can observe that with a reduced $\gamma_{1}$ value, only 4 voice flows are admitted. When no admission control is used, the throughput of FTP flows goes to zero. This is the well-known starvation problem in 802.11e EDCA [36]. When admission control is used, FTP flows avoid starvation from excessive competition from higher priority flows. The value of the RTT (round trip time) for TCP packets value fluctuates greatly under no admission control, but is maintained at a stable level by the admission control algorithm. Thus, our algorithm can protect the performance of the existing best effort traffic flows.

We can further observe that the fourth voice flow at time $50 \mathrm{~s}$ is rejected, but the fifth voice flow at time $60 \mathrm{~s}$ is accepted. This is due to the location-dependent contention in the multi-hop WAN. The fourth flow originates from node 5 which is farther away from QAP than the source of the fifth flow which is node 7. As a result, it causes more contention than the fifth flow and is rejected. This shows the capability of our proposed admission control algorithm to accurately predict the location-dependent contention and maintain QoS while maximizing the spatial reuse of the network.

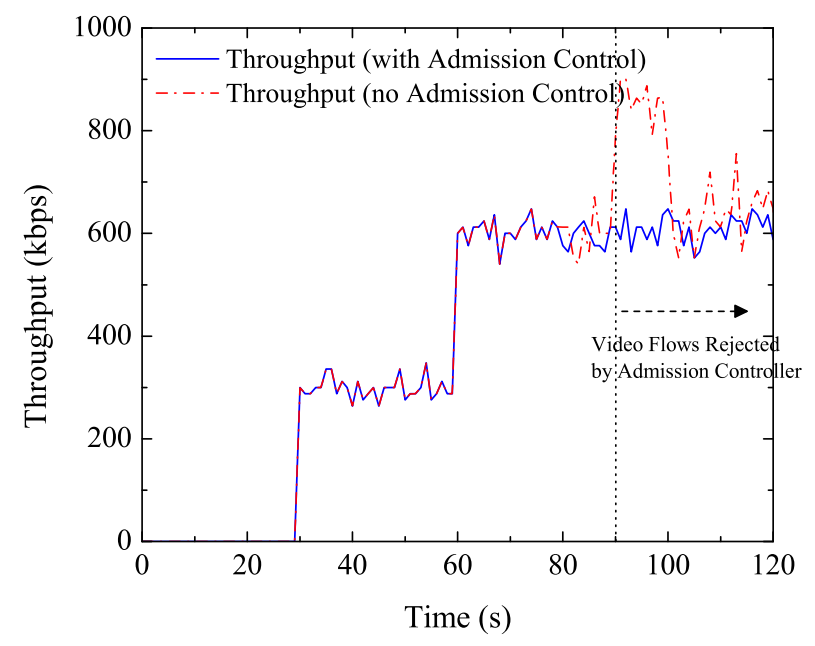

(a) Throughput.

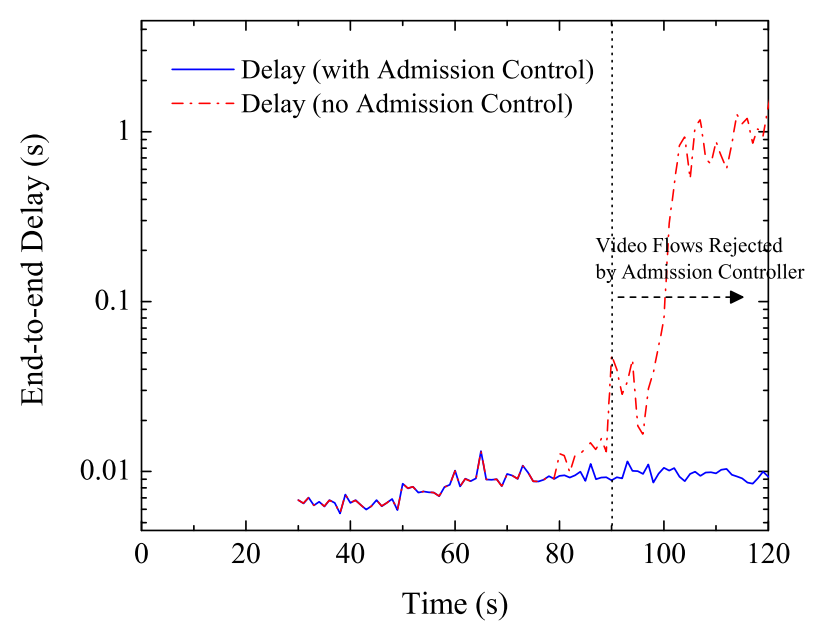

(b) Delay.

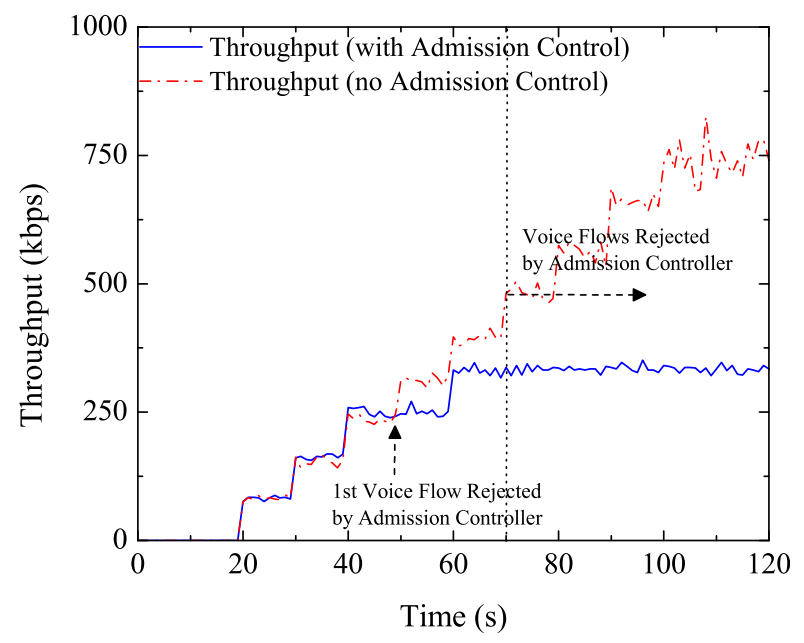

(a) Throughput.

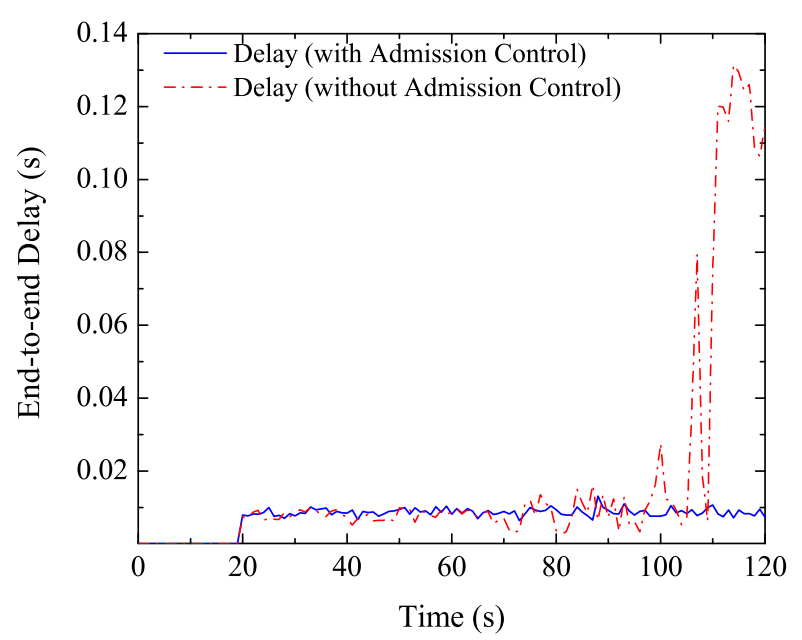

(b) Delay.

Fig. 8. Performance of video flows under random topology.

Fig. 9. Performance of voice flows with FTP flows under random topology. 


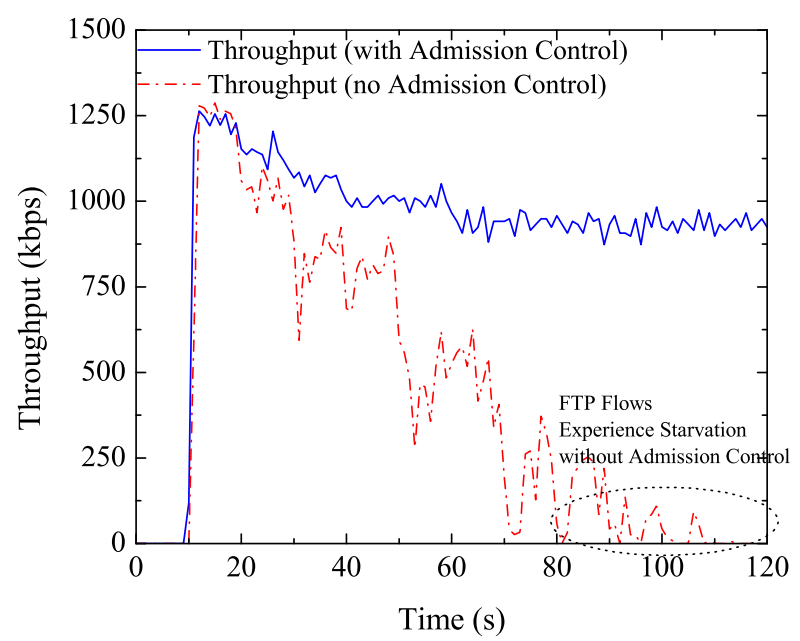

(a) Throughput.



(b) Delay.

Fig. 10. Performance of FTP flows under random topology.

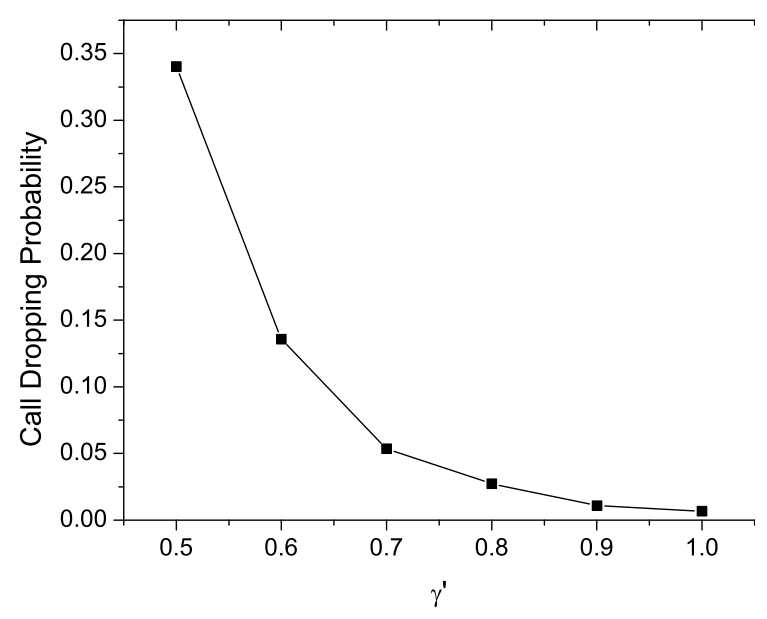

Fig. 11. Call dropping probability with different handoff parameter $\gamma_{i}^{\prime}$.

\subsection{Admission control in a mobile network}

We first investigate the differentiation effects of using $\gamma_{i}^{\prime} \geqslant \gamma_{i}$ for the handoff calls. There are 30 mobile nodes randomly located within a $500 \mathrm{~m} \times 300 \mathrm{~m}$ coverage area. The QAP is located in the middle of the coverage area. All the mobile nodes move according to the random way-point mobility model. The maximum and minimum speeds are 10 and $0 \mathrm{~m} / \mathrm{s}$, respectively. The pause time is $5 \mathrm{~s}$. One third of the mobile nodes are sending $300 \mathrm{kbps}$ video traffic,

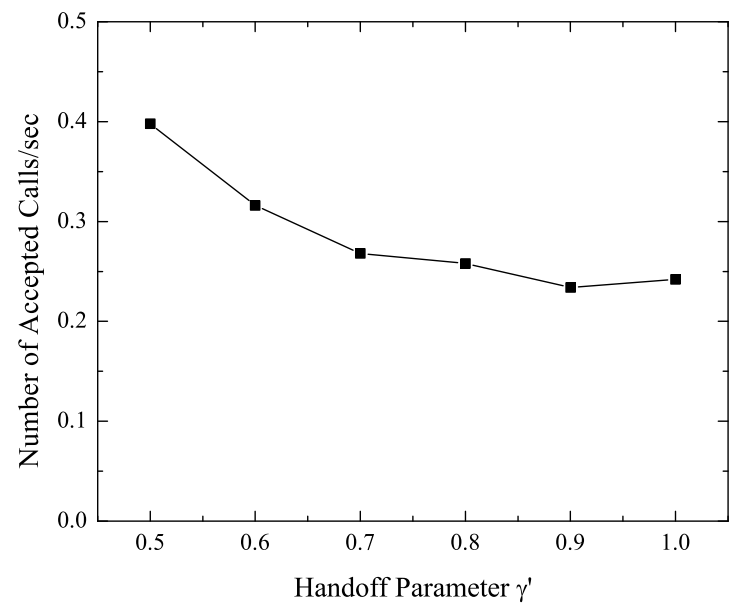

Fig. 12. Average number of received calls per second with different handoff parameter $\gamma_{i}^{\prime}$.

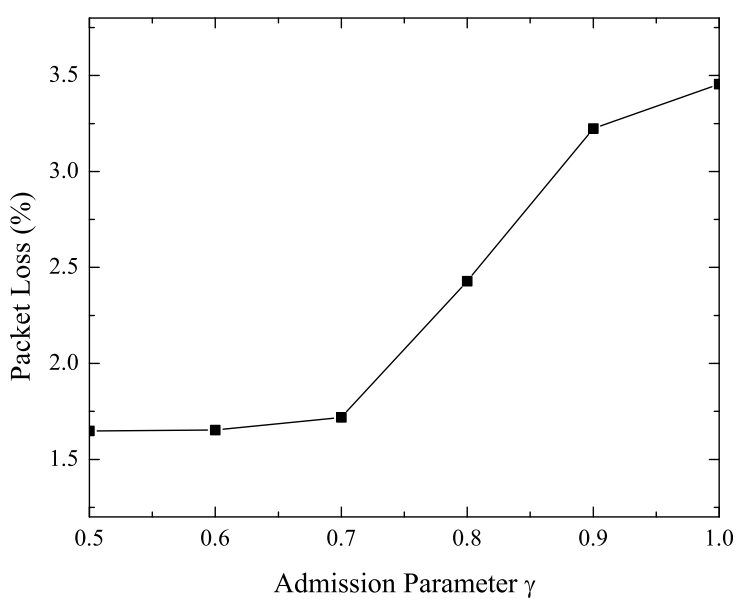

Fig. 13. Packet loss ratio with increasing admission parameter $\gamma$.

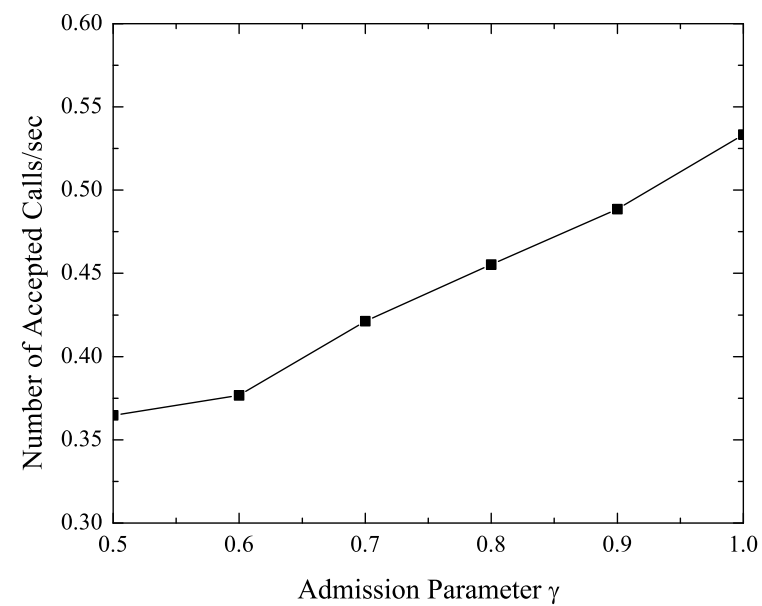

Fig. 14. Average number of received calls per second with increasing admission parameter $\gamma$. 


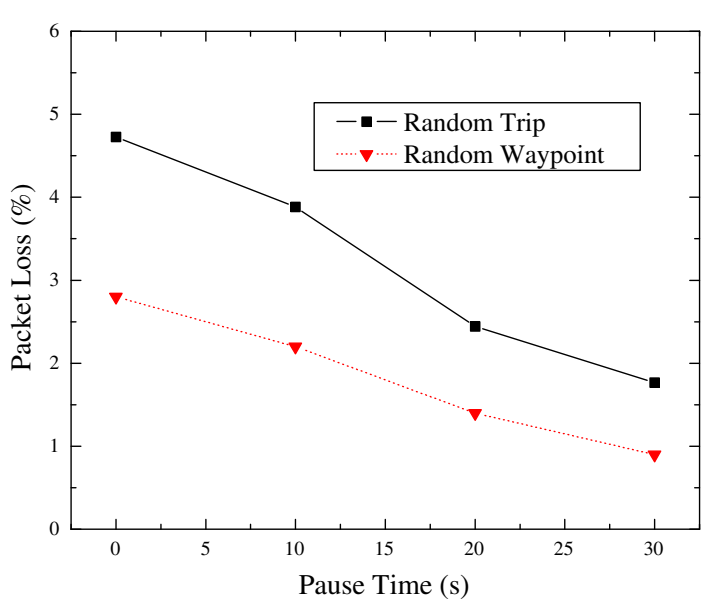

Fig. 15. Packet loss ratio with increasing pause time.

and the other two third of the mobile nodes have $83.2 \mathrm{kbps}$ voice traffic streams as specified in Section 4.1. The Ad hoc On Demand Distance Vector (AODV) routing protocol is used. The call inter-arrival time is exponentially distributed with a mean of $10 \mathrm{~s}$. Call duration is also exponentially distributed with a mean of $15 \mathrm{~s}$. Each simulation runs for $500 \mathrm{~s}$. We fix $\gamma_{1}=\gamma_{2}=0.5$, and vary $\gamma_{1}^{\prime}=\gamma_{2}^{\prime}$ from 0.5 to 1 . The resulting call dropping probability, which is the ratio of the number of dropped calls divided by the total number of ADDTS.renew requests, is shown in Fig. 11. Results show that the call dropping probability is greatly reduced by increasing the difference between $\gamma_{i}$ and $\gamma_{i}^{\prime}$. Fig. 12 shows the average number of new calls accepted per second. We can see that the number of accepted new calls decrease when increased priority is given to handoff calls.

In Figs. 13 and 14, we show the results of packet loss ratio and average number of accepted calls per second when we increase $\gamma_{1}=\gamma_{2}=\gamma_{1}^{\prime}=\gamma_{2}^{\prime}$ from 0.5 to 1 . Each simulation result is the average of three simulation runs when the number of mobile nodes in the network is 20,30 , and 40 , respectively. The other simulation parameters remain the same. The results show that there is a trade-off when selecting the $\gamma$ value. A larger $\gamma$ allows more calls to be accepted, but leads to larger packet losses. Fig. 15 shows the impact of pause time of the mobility model on the packet loss ratio. Thirty nodes are used and $\gamma_{1}=\gamma_{2}=0.5, \gamma_{1}^{\prime}=\gamma_{2}^{\prime}=0.7$. It can be observed that as pause time increases, the network packet loss decreases. The random waypiont mobility model is the default mobility model used by ns-2, and has several shortcomings [37]. Although the proposed admission control algorithm is independent of specific mobility models, its effectiveness may be influenced by the mobility scenarios. As a result, we perform additional simulations under the more advanced random trip mobility model [38]. Results show that there is a slightly higher packet loss ratio under the random trip mobility model. However, the loss rate is still under acceptable levels with the proposed admission control mechanism. The decreased packet loss ratio under the random waypoint mobility model tests may be due to the fact that this model tends to put nodes slightly more concentrated into the central area of the simulation topology which can possibly decrease the routing hops and thus increase the delivery reliability.

\section{Conclusions}

In this paper, we proposed an effective admission control algorithm for multi-hop WLANs based on the use of contention graph and the saturation throughput analysis for each maximal clique's capacity estimation. Extensive simulation tests under different topologies have shown that our proposed admission control algorithm is effective in evaluating the contention status in a multihop WLAN, and makes accurate admission control decisions to prevent the network from congestion. In addition, it can provide QoS guarantee to the existing voice and video flows while maintaining a good performance for best effort traffic. Node mobility is also considered in the model. Simulation results show that the admission control algorithm performs well under a multi-hop WLAN with mobility, and provides a mechanism to differentiate between handoff and new calls. Our proposed admission control algorithm required the complete network topology information at the QAP which makes it a centralized scheme. For future work, we plan to design a distributed algorithm based on limited local information sharing.

\section{Acknowledgment}

This work is supported by the Natural Sciences and Engineering Research Council of Canada under Grant No. 261604-07.

\section{References}

[1] Y. Lin, V.W.S. Wong, M. Cheung, An admission control algorithm for multi-hop 802.11e based WLANs, in: Proceedings of Third International Conference on Quality of Service in Heterogeneous Wired/Wireless Networks (QShine), Waterloo, Canada, August 2006.

[2] IEEE 802.11 WG, IEEE Std 802.11e-2005 (Amendment to IEEE Std 802.11, 1999 Edition (Reaff 2003)), September 2005.

[3] Y. Xiao, H. Li, S. Choi, Protection and guarantee for voice and video traffic in IEEE 802.11e wireless LANs, in: Proceedings of IEEE INFOCOM'04, Hong Kong, China, March 2004.

[4] S.M. Faccin, C. Wijing, J. Kneckt, A. Damle, Mesh WLAN networks: concept and system design, IEEE Wireless Commun. Mag. 13 (2) (2006) 10-17.

[5] O. Oyman, J.N. Laneman, S. Sandhu, Multihop relaying for broadband wireless mesh networks: from theory to practice, IEEE Commun. Mag. 45 (11) (2007) 116-122.

[6] R. Pabst, B. Walke, D. Schultz, P. Herhold, H. Yanikomeroglu, S. Mukherjee, H. Viswanathan, M. Lott, W. Zirwas, M. Dohler, H. Aghvami, D. Falconer, G. Fettweis, Relay-based deployment concepts for wireless and mobile broadband radio, IEEE Commun. Mag. 42 (9) (2004) 80-89.

[7] M.H. Ahmed, Call admission control in wireless networks: a comprehensive survey, IEEE Commun. Surv. Tutor. 7 (1) (2005) 50-69.

[8] D. Gu, J. Zhang, A new measurement-based admission control method for IEEE 802.11 wireless local area networks, in: Proceedings of IEEE PIMRC'03, Beijing, China, September 2003.

[9] D. Pong, T. Moors, Call admission control for IEEE 802.11 contention access mechanism, in: Proceedings of IEEE Globecom'03, San Francisco, CA, December 2003.

[10] Y.-L. Kuo, C. Lu, E. Wu, G. Chen, An admission control strategy for differentiated service in IEEE 802.11, in: Proceedings of IEEE Globecom'03, San Francisco, CA, December 2003.

[11] Z. Kong, D. Tsang, B. Bensaou, Measurement-assisted model-based call admission control for IEEE 802.11e WLAN contention-based channel access, in: Proceedings of IEEE Workshop on Local and Metropolitan Area Networks, San Francisco, CA, April 2004.

[12] G. Bianchi, Performance analysis of the IEEE 802.11 distributed coordination function, IEEE J. Select. Areas Commun. 18 (3) (2000) 535-547.

[13] J. Zhu, A.O. Fapojuwo, A new call admission control method for providing desired throughput and delay performance in IEEE802.11e wireless LANs, IEEE Trans. Wireless Commun. 6 (2) (2007) 701-709.

[14] Y.-J. Choi, S. Bahk, Feedback-based bandwidth allocation with call admission control for providing delay guarantees in IEEE 802.11e networks, Comput. Commun. 28 (3) (2005) 325-337.

[15] Y. Xiao, H. Li, Local data control and admission control for QoS support in wireless ad hoc networks, IEEE Trans. Veh. Technol. 53 (5) (2004) 15581572.

[16] D. Niyato, E. Hossain, Radio resource management in MIMO-OFDM-based wireless infrastructure mesh networks: issues and approaches, IEEE Commun. Mag. 45 (11) (2007) 100-107.

[17] Y. Yang, R. Kravets, Contention-aware admission control for ad hoc networks, IEEE Trans. Mobile Comput. 4 (4) (2005) 363-377. July.

[18] H. Wei, K. Kim, A. Kashyap, S. Ganguly, On admission of VoIP calls over wireless mesh network, in: Proceedings of IEEE ICC'06, Istanbul, Turkey, June 2006.

[19] K. Nahm, A. Helmy, C.-C.J. Kuo, TCP over multihop 802.11 networks: issues and performance enhancement, in: Proceedings of ACM MobiHoc'05, May 2005.

[20] A. Acharya, A. Misra, S. Bansal, High-performance architectures for IP-based multihop 802.11 networks, IEEE Wireless Commun. Mag. 10 (5) (2003) 22-28. Oct. 
[21] P.C. Ng, S.C. Liew, Throughput analysis of IEEE802.11 multi-hop ad hoc networks, IEEE/ACM Trans. Netw. 15 (2) (2007) 309-322.

[22] K. Wang, F. Yang, Q. Zhang, Y. Xu, Modeling path capacity in multi-hop IEEE 802.11 networks for QoS services, IEEE Trans. Wireless Commun. 6 (2) (2007) 738-749.

[23] G. Mergen, L. Tong, Stability and capacity of regular wireless networks, IEEE Trans. Inform. Theory 51 (6) (2005) 1938-1953.

[24] P. Gupta, P. Kumar, The capacity of wireless networks, IEEE Trans. Inform. Theory 46 (2) (2000) 288-404.

[25] A. Zemlianov, G. de Veciana, Capacity of ad hoc wireless networks with infrastructure support, IEEE J. Select. Areas Commun. 23 (3) (2005) 657667.

[26] M. Kodialam, T. Nandagopal, Characterizing achievable rates in multi-hop wireless mesh networks with orthogonal channels, IEEE/ACM Trans. Netw. 13 (4) (2005) 868-880. Aug.

[27] J. Hui and M. Devetsikiotis, Performance analysis of IEEE 802.11e EDCA by a unified model, in: Proceedings of IEEE Globecom'04, Dallas, Texas, November 2004.

[28] J.W. Robinson, T.S. Randhawa, Saturation throughput analysis of IEEE 802.11e enhanced distributed coordination function, IEEE J. Select. Areas Commun. 22 (5) (2004) 917-928. June.

[29] Z. Kong, D. Tsang, B. Bensaou, Performance analysis of IEEE 802.11e contention-based channel access, IEEE J. Select. Areas Commun. 22 (10) (2004) 2095-2106.
[30] Y. Xiao, Performance analysis of IEEE 802.11e EDCF under saturation condition, in: Proceedings of IEEE ICC'04, Paris, France, June 2004.

[31] Y. Lin, V.W.S. Wong, Saturation throughput of IEEE 802.11e EDCA based on mean value analysis, in: Proceedings of IEEE WCNC'06, Las Vegas, Nevada, April 2006.

[32] Y. Chen, J.-A. Francisco, W. Trappe, R. Martin, A practical approach to landmark deployment for indoor localization, in: Proceedings of IEEE SECON'06, Reston, VA, September 2006

[33] J. Yin, Q. Yang, L.M. Ni, Learning adaptive temporal radio maps for signalstrength-based location estimation, IEEE Trans. Mobile Comput. 7 (7) (2008) 869-883.

34] http://www.isi.edu/nsnam/ns/. ns-2 simulator.

[35] C. Perkins, P. Bhagwat, Highly dynamic destination-sequenced distance-vector routing (DSDV) for mobile computer, in: Proceedings of ACM SIGCOMM'94 London, UK, September 1994.

[36] N. Ramos, D. Panigrahi, S. Dey, Quality of service provisioning in $802.11 \mathrm{e}$ networks: challenges, approaches, and future directions, IEEE Netw. 19 (4) (2005) 14-20. July/Aug.

[37] C. Bettstetter, G. Resta, P. Santi, The node distribution of the random waypoin mobility model for wireless ad hoc networks, IEEE Trans. Mobile Comput. 2 (3) (2003) 257-269. July-September.

[38] J.-Y. Le Boudec, M. Vojnovic, The random trip model: stability, stationary regime, and perfect simulation, IEEE/ACM Trans. Netw. 14 (6) (2006) 11531166. December. 